\title{
Dizionarietto goldoniano
}

\author{
Paolo Puppa
}

AMBigUiTA'. Ambigua, la riforma goldoniana, all'insegna di un compromesso inevitabile. La scena aperta verso il mondo, tesa a imitare il reale e il presente della sala, deve convivere colle convenzioni e colle pigrizie del palcoscenico. Il percorso che dagli scenari e dalle maschere conduce ai capolavori della maturità, ai Rusteghi, alla Casa nova, alla Trilogia per la Villeggiatura si incrocia colle resistenze del mercato culturale e coll'articolazione dei gusti e dei modelli dell'epoca. Ogni teatro è associato infatti ad un determinato repertorio, e specie tra il San Samuele, il Sant'Angelo e il San Luca riverberano libelli, gazzette, denunce, con capocomici contesi dall'uno all'altro a suon di ingaggi. Non di una evoluzione lineare, pertanto, si tratta, ma di una convivenza incessante, che crea smottamenti, equilibri e contraddizioni tra vecchio e nuovo entro la medesima drammaturgia. Insomma, la strategia dello specchio e quella della lanterna magica si coniugano tra di loro.

MUSICA. Sono, innanzitutto, ragioni di geografia teatrale. Negli anni della riforma, sono di fatto più le sale in cui si canta che quello dove si dialoga. L'audience privilegia drammi giocosi e melodrammi, e la nobiltà imprenditoriale investe in conservatori e in accademie musicali. Anche se ne L'Impresario delle Smirne del 1760 ne fa una feroce parodia grazie allo sguardo straniero del turco Alì, Goldoni sforna di continuo arie e recitativi, mentre gli infiniti libretti composti per musica influenzano pure i duetti dialogici, i concertati, il ritmo coreutico della conversazione. E se nel copione- manifesto della riforma, Il teatro comico del 1750 la cantatrice Eleonora viene umiliata nella leggerezza della dizione e nella fatuità d'interprete, nondimeno pathos e tenerezze illanguidiscono spesso gli innamorati del commediagrafo veneziano. E l'opera lirica produce un clima fiabesco, determina l'elogio dell'inverosimile, implica le fantasticherie al potere. Anche prima del trentennio parigino in cui si rassegna agli scenari del Théatre des Italiens, proprio nella fase che vede costoro fondersi coll'Opéra comique, pure in precedenza, cioè fin dal periodo del San Luca, Goldoni contende colle sue spose persiane, colle peruviane e colle belle selvagge ai rivali Gozzi e Chiari il monopolio del meraviglioso! 
COMPAGNIA. Altra fonte di ambiguità e di compromessi. Il poeta sequestrato dalla troupe, accerchiato dalla gerarchia dei ruoli, condizionato dalla grammatica delle parti. I vecchi e gli zanni, i padroni e i servi omologati nell'uso della maschera, e poi i giovani amorosi col codazzo di coppie e di doppi, e di servette disinvolte, tutta una memoria di stereotipi di trame, di zibaldoni, di gag e di funambolismi verbali e fisici, l'eredità della commedia dell'arte, ossia un raffinato artigianato industriale che per due secoli ha girato e s'è imposto nelle corti e nelle piazze di tutta Europa. Questo il destino dell'avvocato. Ma in ogni caso, là dove sussiste la maschera, bisogna procedere con cautela, stendere le battute premeditate a poco a poco, sostituendole a quella improvvise, scelte dagli attori. Dunque, si può affermare che il testo goldoniano nasce anche all'incrocio tra il canto e l'acrobazia, tra un suono e un'immagine ginnica che si contendono il primato nella percezione della sala. Certo, Goldoni si ripromette di rieducare la troupe teatrale che gli commissiona di volta in volta i copioni, smascherandola alla lettera, ridando cioè un volto individuale ai personaggi. $\mathrm{E}$ coll'anima psicologica, di memoria cartesiana, un copione sempre più scritto, da imparare. Da qui, la ridda di mogli buone e sagge, di padri di famiglia e di figlie obbedienti, di madri amorose, tra finali rassicuranti che puntualmente suggellano veri e propri inferni domestici. Da qui, la riabilitazione del mercante Pantolone, tolto ai pruriti erotici che lo distraevano negli scenari secenteschi, e trasformato in spartitraffico di equilibrio sociale e di armonia coniugale, specie nel periodo del Sant'Angelo. Ma nel frattempo, il commediografo sequestrato dalla compagnia rovescia la costrizione in vantaggi, in stimoli costanti per la scrittura stessa, quasi una metrica per la versificazione. Al di là del bon ton, quante bizzarrie in effetti emergono dalla convivenza cogli attori, quale formidabile concentrato di vizi e di nevrosi entro la gerarchia della compagnia stessa, con rotture brusche e ritorni inopinati di attori, feroci gelosie professionale, coll'avvocato piccolo Casanova che colleziona cuori infranti tra le attrici, specie se servette! Quale osservatorio del mondo, garantito da dietro le quinte!

ServetTA. Le Baccherini, le Marliani, le Bresciani, etc. Tutte interpreti dotate di inventiva, adatte a metamorfosi frenetiche, a giochi di seduzioni e di travestimenti, esaltando la macchina teatrale, quasi portavoce dell'autore nel suo portar avanti la storia. In una parola, donne di spirito contrapposte nel linguaggio del commediografo alle donne dei vapori, ossia alle prime attrici, alle Rosaure amorose, abituate a spadroneggiare nella troupe grazie alle peripezie della passione. Le Smeraldine, le Coralline, le Argentine, le Mirandoline, e dunque le Cameriere brillanti, le Serve amorose, le Castalde, le Locandiere, tolte agli intermezzi comici di Colombina, ai lazzi coi servi cenciosi nei sottoscala degli scenari a parodiare le svenevolezze delle padroncine, vengono adesso riabilitate dall'avvocato, nominate sul campo protagoniste, al punto da sfiorare negli intrecci nozze clamorose coi loro datori di lavoro, o da sbaragliare finte 
misogenie nella controparte maschile. Questione di pochi anni. La loro rivolta verrà rintuzzata, se già nelle Massere del 1754 le rivediamo in gruppo, declassate e rese innocue nel loro muoversi in massa, alla ricerca di avventure carnevalesche. Ma intanto, proprio da queste suivantes, nel gergo di Marivaux, cioè confidenti, e dal ruolo della brillante nella compagnia goldoniana sprigioneranno in seguito i lustrini e le paillettes della soubrette nel varietà novecentesco...

CARNEVALE. Ben 13, nella drammaturgia goldoniana, sono le commedie che ospitano il carnevale, o meglio che debuttano a chiusura del detto periodo festivo. Da La putta onorata del 1749 a Chi la fa l'aspetta del 1764, il morbin, i chiassetti, gli spassi, le amene licenziosità esaltate dai balli e dalle maschere, incalzano dalla strada, bussano alla porta di case tristi o parsimoniose, creano un vociante teatro fuori dai palcoscenici deputati. I rusteghi stessi, così refrattari ai palchi, così inibiti allo spettacolo, non si accorgono che lo spettacolo della finzione sarà allestito proprio al di qua delle loro porte inutilmente sbarrate. Così, una non resistibile voglia di incontri e di occasioni amorose, in un turbinio di gemellaggi e di scambi di identità assale Momoli scapestrati e Anzoletti disponibili, garzoni e vecchi avari, acerbe giovinette e decrepite in fregola, le Roseghe massere e le Silvestre Morbinose, queste ultime interpretate da attori maschili secondo le regole del travestismo antico.

CENSURA. Ma c'è, oltre al resto, una severissima magistratura che controlla gli spettacoli. Sulle scene della Serenissima non è consentito ai servi basto nare i padroni, a donne sole mostrarsi in strada o a preti infilarsi negli intrecci. La licenziosità e il libertinaggio, praticati nei palchi e nei ridotti, sono viceversa banditi dal palcoscenico, anche nel clima di riforma morale che soffia nel tempo dell'Encyclopédie. Così, guai alla mésaillance, vale a dire il matrimonio tra aristrocratici e borghesi. E l'avvocato Goldoni, se nel 1750 rimaneggia la Pamela di Samuel Richardson, consentendo alla virtuosa protagonista le nozze col nobiluomo, solo dopo che s'è scoperto sangue blu nelle vene della fanciulla, lo fa non per la natura moderata del suo illuminismo (e dei ceti intellettuali veneti in generale), ma per specifiche leggi istituzionali che sanciscono la cancellazione dei nobili, sposati con donne borghesi, dal cursus honorum...

LUOGHI. Altra forma di censura, l'obbligo di collocare in altre città perso naggi nobili, qualora viziosi! Così le Palermo, le Napoli, le Livorno, a dispiegar una araldica di fantasia. Ma luoghi oltre le calli e i campielli veneziani, oltre i salotti della Serenissima non son dettati solo dalle sanzioni della Magistratura. Tutta l'Italia, al di là del labirinto lagunare, diviene un albergo di libero scambio, un'inifita locanda, un contrassegno di spostamenti autobiografici e simbolici per il commediografo viaggiatore, un'occasione socializzante per confronti antropologici. Può essere 
l'Inghilterra dei filosofi amati, o la Francia e la serie di etnie nazionali contrapposte alla nostra nell'utopia del civile confronto cosmopolita. E non manca l'antico, i luoghi della biblioteca ideale, la Ferrara del Tasso o la Roma di Terenzio o la stessa Parigi di Molière, là dove il commediografo sospende la sua riforma, coll'angoscia dell'effimero che potrebbe subentrare, e si volge indietro verso l'alloro del poeta incoronato, cui l'avvicinano tribolazioni da parte dei nemici e vapori malinconico-depressivi. E ancora l'esotico, l'oltre nello spazio, più che nel tempo. Tutte contraddizioni rispetto alla priorità dello specchio, ad una strategia, ripeto, in cui la scena adegua spazi e tempi sulla misura della sala.

ARLECCHINO. Ultima maschera a sparire (sarà un editto della Milano cisalpi na del 1801 a decretare la fine delle maschere tutte dai nostri palcoscenici), è Arlecchino a intrattenere con Goldoni un oscuro legame di rivalità feroci e di funzionali alleanze. Zanni disceso dalle valli bergamasche, e prima ancora forse uscito da un sottosuolo demoniaco o dai riti di fecondazione, attestato dalla screziatura cromatica dell'abito, eroe goffo e gaglioffo negli scenari di due secoli, immortalato nell'immaginario del pubblico europeo dai virili contorsionismi dei Martinelli o dalle eleganze rococò dei Visentini, pur sotto una proliferante varietà di nomi, da Truffaldino a Mezzettino a Traccagnino, etc., Arlecchino si impone all'avvocato, lo stana dalla carte forensi di Pisa negli anni' 40 incarnandosi nelle giullarate sublimi di Antonio Sacchi, e lo accoglie profugo a Parigi negli anni'60 effigiandosi nella grazia e nella leggiadria del Carlin Bertinazzi. Togliergli la maschera, magari mostrarlo nei panni d'un moro, d'un africano, come nelle Femmine puntigliose del 1750, ne accentua solo la diversità. Sotto la maschera, infatti, non c'è che un volto anonimo. Sotto il simulacro, non traspare la psicologia d'un individuo autonomo, ma la fame, la paura, la rabbia d'un imbroglione disperato e rabbioso, fonte di disagio più che di riso per la sala illuminista.

LINGUA. Servette e Arlecchini, nobili e borghesi, e una gamma vastissima di diversi mestieri, come si esprimono? In dialetto, di solito, un dialetto però non più veicolo di subalternità culturale, oggetto di scherno, associato a caricature umane, quanto piuttosto dignificato, innalzato a contesti molto articolati e dalle infinite sfumature esistenziali. E questo dialetto sale fino ad incontrarsi con un italiano non pedantesco, a sua volta abbassatosi di livello lessicale-sintattico, mentre una terza lingua, la francese, sta all'orizzonte. Si crea in tal modo una sorta di casa per i parlanti, citando la bella metafora di Folena, dove ogni piano, da quello a terra al mezzanino al piano nobile corrisponde ad uno di questi codici linguistici, tra i quali pia ni il personaggio si sposta altresì di continuo a seconda e del suo interlocutore e della propria condizione emotiva. Ma attenzione! Se dal mondo alla rovescia, caro al carnevale, e dalla tradizione delle maschere persiste la comunicazione sfasata tra parlanti idiomi diversi, con bisticci, alterazioni 
di senso, ipercorrettismi con effetti parodici, insomma la babele linguistica degli scenari, Goldoni recupera tale ingrediente comico depurandolo e motivandolo. Le «stroppiature» vengono rese più sobrie, come annuncia il capocomico Orazio nel Teatro comico, margari agganciate ad un intento moralistico: ad esempio, l'Isidoro levantino nelle Donne di casa soa del 1755 che si esprime in un veneziano maccheronico per lanciar anatemi nemmeno tanto inverosimili contro le donne italiane spendaccione e ipocrite. E, del resto, la pur esplicita tendenza all'omologazione non evita, anzi sviluppa quel ritmo straordinario di concertato, il miracoloso intarsio di entrate ed uscite dei protagonisti dalla battuta. Qui van ribaditi i rimandi di tale ariosità oltre che dalla musica contigua, anche dalle tecniche all'improvviso, annullate da Goldoni, ma innestate indirettamente nel montaggio del testo. Paradossalmente, dagli idilli popolani, dal Campiello e dalle Baruffe, nasce più di uno stimolo e di situazioni e di atmosfere linguistiche per la mediazione nazionale che attraverso i Promessi sposi manzoniani tende a inglobare l'oralità in un italiano non letterario.

MEMORIE. Iniziati nel 1783, a quasi ottantanni anni nel soggiorno parigino, editi quattro anni dopo in tre tomi, i Mémoires costituiscono una parziale, lacunosa ricostruzione della propria tumultuosa, nomadica esistenza. Un'affabulazione, un filò, una bugia colorata, non solo per l'intento agiografico e gnomico che consegna una vocazione teatrale ai posteri, ma sopratutto per i registri da romanzo settecentesco, che vi mescola le tecniche e le formule più astute in voga entro l'industria culturale della fiction nell'epoca dei Lumi, dalla commedia lagrimosa all'intreccio libertino al romanzo di formazione (il Bildungsroman), sicché se la carretta dei comici non l'avesse distratto, chissà che De Foe o Fielding avremmo avuto in Laguna! 